\title{
Counting the Number of Shortest Chamfer Paths in the Square Grid
}

\author{
Laith Alzboon, Bashar Khassawneh, Benedek Nagy \\ Department of Mathematics, Eastern Mediterranean University, Famagusta, \\ North Cyprus, via Mersin-10, Turkey, Laith.Khalaf@emu.edu.tr, \\ bashar.khassawneh@emu.edu.tr, benedek.nagy@emu.edu.tr
}

\begin{abstract}
In this paper, the number of shortest paths between any point pairs of the square grid, based on weighted distances, is computed. We use two types of steps on the gridlines and diagonal steps. Consequently, we use an 8-adjacency square grid, that is, one where a first weight is associated with the horizontal and vertical movements, while a second weight (not necessarily different from the first) is assigned to the diagonal steps. The chamfer distance of two points depends on the numbers and weights of the steps in a 'shortest path'. In special cases, the cityblock and the chessboard distances, the two most basic and widely used digital distances (they are also referred as $L_{1}$ and $L_{\infty}$ distances, respectively) of the two-dimensional digital space occur. Although our combinatorial result is theoretical, it is closely connected to applications, such as communication networks, path counting in digital images, traces and trajectories in $2 D$ digital grids. We consider all seven cases with non-negative weights and also the case when negative weights are allowed.
\end{abstract}

Keywords: traces; trajectories; weighted distances; shortest paths; digital distances; combinatorics

\section{Introduction}

In this paper we are interested in answering a combinatorial question about the number of cheapest solutions of a problem. More precisely, a solution is considered as a path between two grid points. The number of cheapest solutions is, then, the number of minimal weighted paths between the points.

Digital grids and their applications in various fields play important roles in science and technology. Digital grids are used in applications such as image processing [11], computer graphics, communication networks, crystallography and physical simulations. The space, in this case, the considered grid, is discrete, so theoretic tools from discrete mathematics, graph theory, combinatorics and, especially, from digital geometry can be used. In most cases only coordinates with integer values are used to address points (nodes). The square grid (also called rectangular 
grid) is the most usual digital grid, as it is the most frequently applied grid in two dimensions (2D). It is essential in image processing, cellular automata and other fields of applied information technology as well as 2D physical simulations. The Cartesian coordinate frame describes it, and, consequently, two well-known neighbourhood relations are defined based on those coordinate values. One of the benefits of working on the square grid is that it is self-dual: the square grid can be seen by connecting (the midpoints of) neighbour pixels to each other, and also by using the original grid, with points where the grid lines cross. In discrete grids, neighbour relation is of high importance. Opposite to discrete space, Euclidean space is continuous space, and there is no neighbour relation. The natural, Euclidean distance has several well-known and beneficial properties. However, using Euclidean distance on a discrete space may not be the best option. For example, one topological paradox is that the grid points having an exact Euclidean distance of seven from the origin do not really form a circle in any usual sense; the determined four pixels are not even connected. When working with computers, one may prefer digital distances, i.e., distances based on paths through neighbour points. In most cases, these distances have integer values, and it is easy to work with them. The shortest paths between any two points are computed depending on the types of the steps of the paths: they could be horizontal, vertical or diagonal between any two adjacent points. In this context, movements to 4- and 8neighbourhoods are defined. In discrete spaces, especially in the square grid, digital distances, have been proposed in [18]. Two basic distance functions are defined based on the two neighbour relations. The cityblock (also called the Manhattan taxicab) distance and the chessboard distance are related to the 4- and 8-neighbourhood movements, respectively. Since these two digital distances give very rough approximations of the Euclidean distance, it was recommended to use them alternating along a path (the obtained distance is called octagonal distance). From the end of 1980's, extending this idea more formally, digital distances based on predefined neighbourhood sequences have been introduced and used in which both chessboard and cityblock neighbourhoods are combined in a sequence that can be periodic [19, 4, 5] or non-periodic [14]. Distances based on neighbourhood sequences on other grids have also been defined, see, e.g., [13]. Other digital distances, the weighted distances give another way to have distances on a grid with integer values [2]. They are also called chamfer distances. Given the associated weights for the used neighbourhoods, the chamfer distance between $p$ and $q$ relative to these weights is the length (i.e. the sum of the weights) of the shortest digital arc (path) from $p$ to $q$ with respect to the weights of movements. One of the advantages of weighted distances is that one can approximate the Euclidean distance by them in a smooth way (especially allowing larger steps and more weights). Another reason to prefer weighted distances versus distances based on neighbourhood sequences is that the former ones are always metric, while there are plenty of neighbourhood sequences that do not provide metrics [14] (since the triangular inequality may fail). 
Counting the number of shortest paths is a theoretical, combinatorial problem which has various connections for applied fields. The number of the cheapest solutions may give significant information about the problem to be solved. In networks, communication-related fields, computer simulations and in theory of algorithms some concepts and algorithms from graph theory play important roles. In communication networks the transmitters, receivers, etc. can be represented by nodes of a graph, and their connections, the possible ways of communication, can be shown by the edges. Concepts such as paths, shortest paths and distances in these graphs are understood and give some important features of the communication network. The number of shortest paths also has importance in applications for transmitting messages over networks since they refer to the width of the connection channel between the given points. Any shortest path can be useful and used to increase the performance, in this case, the amount of information transmitted during a unit of time (the width of the network), speeding up communication [3]. Opposite to the Euclidean space, the shortest path is usually not unique over discrete spaces (i.e., there is no unique cheapest solution). These networks are usually artificial, meaning that graphs with special properties can be considered, such as the square grid. In social networks, the various graph measures, such as eccentricity, are defined based on the number of some shortest paths [8]. In some physical simulations connected to random walks, percolations, trajectories and traces $[9,10,17]$, it is also important to count the number of shortest paths. Several related applications have already been detailed in [12]. Path counting in discrete spaces is closely related to graph theory. It is well-known that the number of paths with a given length can be computed by the appropriate powers of the adjacency matrix of the graph. Path counting (for cityblock and chessboard distances) in digital images (i.e., finite subgraphs of the square grid) is used to infer properties of images [18]. It was also considered in [7] based on matrix multiplication with various neighbourhood relations. In [6], the numbers of shortest paths are computed for the two above mentioned basic distances and also to the octagonal distance, which is a special neighborhood sequence-based distance. For neighbourhood sequences in general, the problem was considered in [15]. In this paper, a similar combinatorial problem, the path counting for weighted distances considering the basic two types of steps is presented with enumerative combinatorial calculation. In most cases, we assume that both weights are non-negative. Moreover, we solve all the cases of the problem by providing the solutions by closed formulae. As we will see that there are five entirely different cases based on (the relation of) the used weights if both weights are positive; and there are two cases with 0 weights. Two of the cases, actually, provide the same result as the corresponding results for cityblock and chessboard distances, however, our proof technique is different from the technique used in [6]. We also present 3D charts to show how the number of shortest paths grows when the distance grows. Thus, the significance of the paper is not only to consider and summarize all the possible cases, but also to give solutions for cases which were not analyzed before, e.g., the last three cases shown in this paper. Few 
of the cases presented in this paper were already presented in [1]. Those results are complemented here with various results of non-traditional weight settings, e.g., allowing the diagonal steps to have less weight than the cityblock steps, or to have zero or negative weight.

\section{Preliminaries}

Now we recall some definitions and concepts that are necessary to understand the results of this paper. As we briefly mentioned before, there are two popular types of neighbourhood relations in the square grid: the cityblock and the chessboard neighbourhood. The cityblock's four neighbours of a point of the square grid are defined as the edge-adjacent points: two horizontal and two vertical neighbours (see Figure 1, left). The chessboard neighbourhood of a point, in addition to the previously given four points, also contains the four closest points in diagonal directions (see Figure 1, middle). These neighbourhood relations are also called von Neumann and Moore neighbours, respectively, in cellular automata theory.
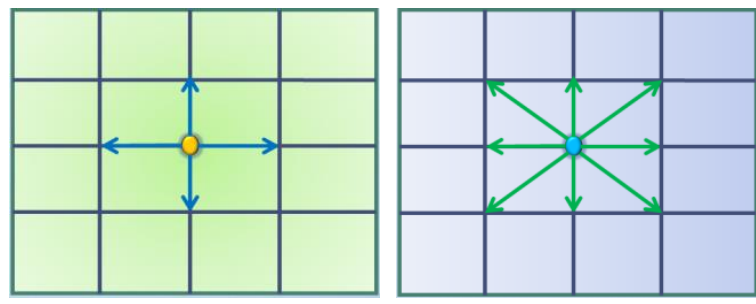

Figure 1

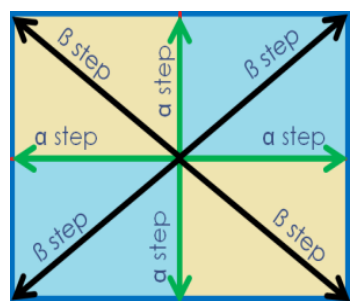

○一⿻上丨

Cityblock neighbours (left, four neighbours for each point). Chessboard neighbours (middle, eight neighbours for each point). Weighted steps for chamfer distance (right, $\alpha$ steps, $\beta$ steps) from the point in the centre.

Weighted distance (or chamfer distance per the original terminology [2]) is used in the square grid to get a better approximation to the Euclidean distance in twodimensional digital space than the distances based on the sole use of the chessboard or cityblock neighbourhood. According to the movements from a point to one of its neighbours, we associate a weight for each type of movement: we give weight $\alpha$ for cityblock movements and weight $\beta$ for each diagonal movement, as shown in Figure 1 (right). Formally, we can describe it as follows:

Let $p=\left(x_{1}, y_{1}\right)$ and $q=\left(x_{2}, y_{2}\right)$ be two points in the square grid; let $W=\left(w_{1}, w_{2}\right)$ be the absolute difference vector between the points, where $w_{1}=\left|x_{1}-x_{2}\right|$ and $w_{2}=\left|y_{1}-y_{2}\right|$. Then, as was previously computed [18], the cityblock distance (Manhattan taxicab distance) can be computed as $d(p, q)=w_{1}+w_{2}$ and the chessboard distance can be computed as $d(p, q)=\max \left\{w_{1}, w_{2}\right\}$. 
The number of diagonal steps in a shortest path of the chessboard distance is $\min \left\{w_{1}, w_{2}\right\}$, and the number of cityblock steps (i.e. the number of vertical or horizontal movements) in a shortest path with the chessboard distance is $\max \left\{w_{1}, w_{2}\right\}-\min \left\{w_{1}, w_{2}\right\}$. These values become important when calculating the number of shortest paths of chamfer distances.

When using both types of neighbours, but with different weights, in order to calculate the length of a shortest path (i.e. the chamfer distance between $p$ and $q$ ), we must find how many $\alpha$ and $\beta$ steps are in the given path. Their numbers in a shortest path depend on the respective values of $\alpha$ and $\beta$ as well. According to their numbers and values, we will compute not only the length but also the number of shortest paths between any two points. The actual computation depends on the used weights. In this paper, as usual, both in graph theory and in digital geometry, we assume that both $\alpha$ and $\beta$ are non-negative, and actually, in the first some cases we assume that they are positive. In some applications, there is also an assumption that $0<\alpha \leq \beta$. We show Example 1 below, for this case. With this condition, subcases are defined by the relation of $2 \alpha$ and $\beta$. However, in this paper, we do not restrict our studies to these cases. We will also do computations when $0<\beta<\alpha$ (see Subsection 3.5), and as we will see this case is the most interesting among all. For the sake of completeness, we also present the cases, when one or both the weights have value 0 .

Now, as an example, we show how to compute the distance, or the length of the shortest path if $0<\alpha \leq \beta$ holds. Let $N$ be the number of $\alpha$ steps and $M$ be the number of $\beta$ steps in a shortest path; then, the weighted distance between $p$ and $q$ is $d_{w}(p, q)=N \alpha+M \beta$.

Example 1. Let $p=(5,6), q=(7,1)$ and $\alpha=3, \beta=4$. Then $w_{1}=2$ and $w_{2}=5$. Thus, the cityblock distance of these points is 7 , their chessboard distance is 5 (note that in these distances unit weight is used). Now, computing the chamfer distance, since $\alpha<\beta<2 \alpha$, it is worth using the path defined by the chessboard distance, i.e. with 2 diagonal and 3 cityblock steps: the chamfer distance equals to: $2 \cdot 4+3 \cdot 3=17$.

\section{Results: Formulae for the Number of Shortest Paths}

According to the values of $\alpha$ and $\beta$, we can compute weighted distances, and, consequently, we can compute the number of shortest paths. In this context, we have various cases depending on the respective ratio of the used weights, letting $f\left(w_{1}, w_{2}\right)$ be the function calculating the number of shortest paths between two points with an absolute difference vector $\left(w_{1}, w_{2}\right)$. The cases are listed in the following subsections. The first two cases are equivalent to obvious discrete mathematical exercises (and have been proven also in [6] by a recursive method), 
and we explain them only for the sake of completeness using enumerative combinatorial techniques in our proofs. In the first five subsections, cases with positive weights are studied, while in the last subsection we deal with the cases when one or both weights is/are zero.

\subsection{Case of $\beta>2 \alpha>0$}

Theorem 1. Let $\alpha$ and $\beta$ be the weights for cityblock and diagonal movements, respectively, such that $\beta>2 \alpha>0$. Let $p=\left(x_{1}, y_{1}\right)$ and $q=\left(x_{2}, y_{2}\right)$ be points of the square grid and $w_{1}=\left|x_{1}-x_{2}\right|$ and $w_{2}=\left|y_{1}-y_{2}\right|$ be the absolute differences between the corresponding coordinates of the points. Then, the number of shortest paths between $p$ and $q$, denoted by $f\left(w_{1}, w_{2}\right)$, is given as $f\left(w_{1}, w_{2}\right)=\left(\begin{array}{c}w_{1}+w_{2} \\ w_{1}\end{array}\right)$.

Proof. We have $\beta>2 \alpha$ such that the weights are positive, which means that in the shortest path between $p$ and $q$, no diagonal steps occur since diagonal steps can be substituted by two consecutive cityblock (i.e. a vertical and a horizontal) steps to produce a path with a smaller weight. Thus, all shortest paths contain only cityblock steps. The number of $\alpha$ steps between points $p$ and $q$ in the shortest weighted paths is computed in the same way as in the cityblock distance: $w_{1}+w_{2}$. The distance between $p$ and $q$ is $\alpha$ times more since each step has weight $\alpha$. Moreover, in each shortest weighted path between $p$ and $q$, the numbers of horizontal and vertical steps are $w_{1}$ and $w_{2}$, respectively. However, the order of these steps is arbitrary; thus, the number of shortest paths is given by the number of ways that we can arrange $w_{1}$ or $w_{2}$ steps among the total $w_{1}+w_{2}$ steps; it is given by the binomial coefficient $\left(\begin{array}{c}w_{1}+w_{2} \\ w_{1}\end{array}\right)$. Actually, $\left(\begin{array}{c}w_{1}+w_{2} \\ w_{1}\end{array}\right)$ and $\left(\begin{array}{c}w_{1}+w_{2} \\ w_{2}\end{array}\right)$ give the same value.

Example 2. The number of shortest paths between the points $p(5,12)$ and $q(8,13)$ with $\alpha=3$ and $\beta=7$ is computed as follows: $w_{1}=|8-5|=3$ and $w_{2}=|13-12|=$ $1, w_{1}+w_{2}=4$. Thus, the result is $f(1,3)=\left(\begin{array}{l}4 \\ 3\end{array}\right)=\left(\begin{array}{l}4 \\ 1\end{array}\right)=4$. See also Figure 2 .

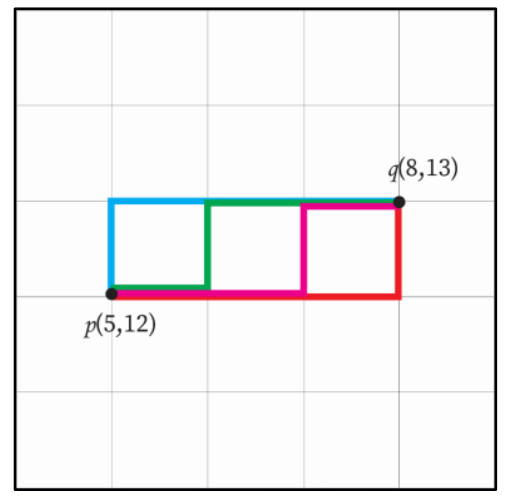

Figure 2

All shortest paths in case $\beta>2 \alpha>0$; the four colors show the four shortest paths of Example 2 
The paths of this case are also called grid-paths since only the edges of the grid are used. Since these results are exactly the binomial coefficients (almost as they form the Pascal's triangle), we do not give them in a table form, only sketch them in Figure 3 as a $3 \mathrm{D}$ chart for the number of shortest weighted paths from point $(0,0)$ to all points in the represented region. In the figure, the origin is placed in the middle to show the symmetry distribution of the values. The formula grows rapidly in the corner directions when the coordinate differences are (almost) equal.

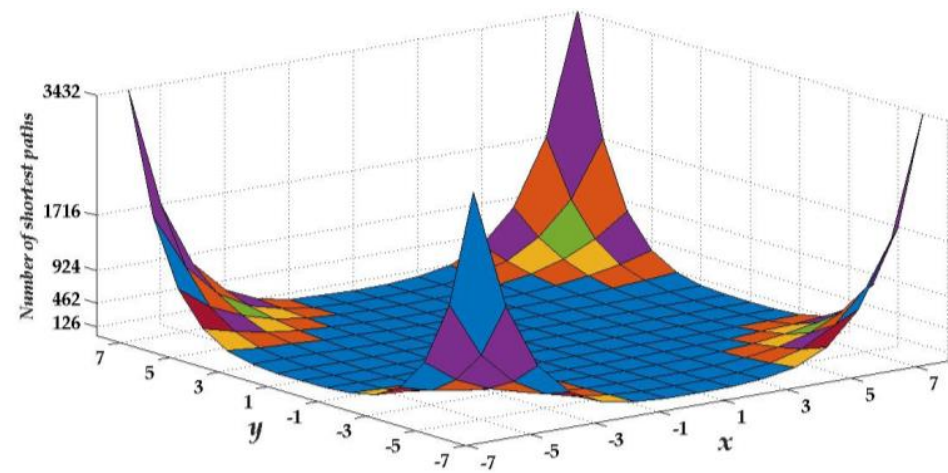

Figure 3

The number of shortest weighted paths from point $(0,0)$ to other points in a $14 \times 14$ window with corners $(-7,-7),(7,-7),(7,7)$ and $(-7,7)$ in case $\beta>2 \alpha>0$.

\subsection{Case $0<\alpha<\beta<2 \alpha$}

Theorem 2. Let $\alpha$ and $\beta$ be the weights for cityblock and diagonal movements, respectively, with the condition $\alpha<\beta<2 \alpha$. Further, let $p=\left(x_{1}, y_{1}\right)$ and $q=\left(x_{2}, y_{2}\right)$ be points, and let $w_{1}=\left|x_{1}-x_{2}\right|$ and $w_{2}=\left|y_{1}-y_{2}\right|$. Then, the number of the shortest paths between $p$ and $q$ is given as $f\left(w_{1}, w_{2}\right)=\left(\begin{array}{c}\max \left\{w_{1}, w_{2}\right\} \\ \min \left\{w_{1}, w_{2}\right\}\end{array}\right)$.

Proof. Both weights are positive and $\alpha<\beta<2 \alpha$. Thus, we may move in the shortest path from $p$ to $q$ using both $\alpha$-steps and $\beta$-steps. We use $\beta$-steps as much as possible to get closer to the endpoint, which means that we will move diagonally by the minimum number of differences between the point coordinates, and the remaining steps are $\alpha$-steps. According to this, the number of $\alpha$-steps and $\beta$-steps in the shortest paths will be computed in the same way, as in a chessboard path from $p$ to $q$ (i.e. the number of steps is $\max \left\{w_{1}, w_{2}\right\}$ ). Since the number of $\beta$ steps is $\min \left\{w_{1}, w_{2}\right\}$, the number of $\alpha$-steps is $\left(\max \left\{w_{1}, w_{2}\right\}-\min \left\{w_{1}, w_{2}\right\}\right)$. The order of the steps is arbitrary; thus, the number of shortest weighted paths equals to the number of ways the $\beta$-steps can be arranged in the path with $\max \left\{w_{1}, w_{2}\right\}$ steps altogether, which is exactly the binomial coefficient $\left(\begin{array}{c}\max \left\{w_{1}, w_{2}\right\} \\ \min \left\{w_{1}, w_{2}\right\}\end{array}\right)$. 
Example 3. Let $p(-2,3), q(2,0)$, and let $\alpha=3, \beta=4$.Then $w_{1}=4, w_{2}=3$,further $\min \left\{w_{1}, w_{2}\right\}=3$ and $\max \left\{w_{1}, w_{2}\right\}=4$. Applying the formula for this case, the number of shortest paths from $p$ to $q$ is $\left(\begin{array}{l}\max \{4,3\} \\ \min \{4,3\}\end{array}\right)=\left(\begin{array}{l}4 \\ 3\end{array}\right)=4$. Actually these four shortest weighted paths are illustrated in Figure 4 with various colors.

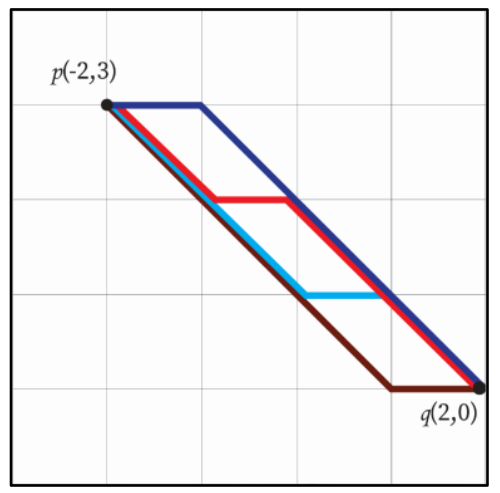

Figure 4

Example for all shortest paths of case $0<\alpha<\beta<2 \alpha$ as in Example 3

Again, the values of Pascal's triangle appear, but in a different arrangement than in the previous case. Figure 5 gives a 3D chart for values for the number of shortest weighted paths from point $(0,0)$ to all points in a $14 \times 14$ window. To show the symmetry of the distribution the origin is in the middle. This graph is already more interesting than the previous one, with more growing directions: the value grows fastest when one of the absolute coordinate differences is (approximately) half of the other one.

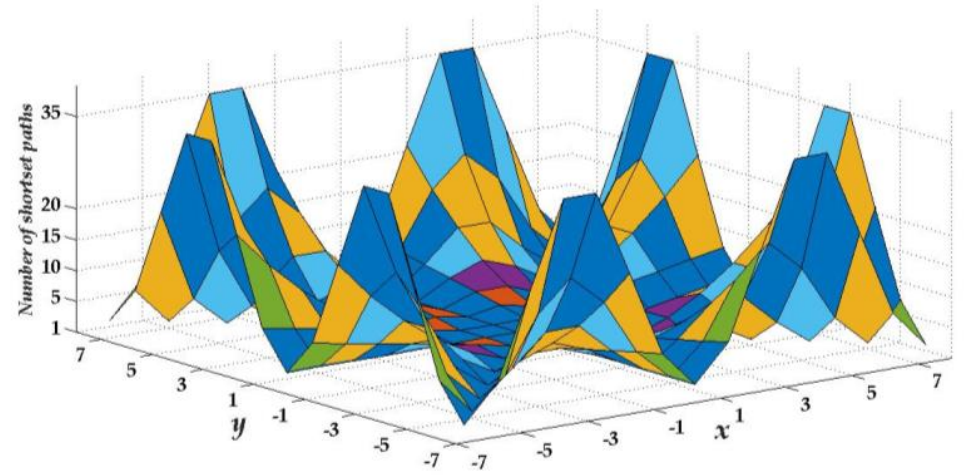

Figure 5

The number of shortest weighted paths in case $0<\alpha<\beta<2 \alpha$ in a $14 \times 14$ window with corners $(-7,-7),(7,-7),(7,7)$ and $(-7,7)$. The minimums on the axes and on the diagonals can be seen. 


\subsection{Case of $\beta=2 \alpha>0$}

Theorem3. Let $\alpha$ and $\beta$ be (positive) weights for cityblock and diagonal movements, respectively, such that $2 \alpha=\beta$. Let $p=\left(x_{1}, y_{1}\right), q=\left(x_{2}, y_{2}\right), w_{1}=\left|x_{1}-x_{2}\right|$ and $w_{2}=\left|y_{1}-y_{2}\right|$. The number of shortest paths $f\left(w_{1}, w_{2}\right)$ between $p$ and $q$ is

$$
f\left(w_{1}, w_{2}\right)=\sum_{i=0}^{\min \{w 1, w 2\}} \frac{\left(w_{1}+w_{2}-i\right) !}{i !\left(w_{1}-i\right) !\left(w_{2}-i\right) !}
$$

Proof. In this case, a diagonal step has exactly the same weight as two consecutive movements to cityblock neighbours. The number of shortest weighted paths between $p$ and $q$ depends on the number of used diagonal steps ( $\beta$-steps) between the two points, which is at most the minimum difference of the two coordinate values of $p$ and $q$. Since each diagonal step can be substituted by two consecutive $\alpha$-steps (a horizontal and a vertical one), the number of diagonal steps may be less, potentially equalling zero, meaning that the points are connected by only cityblock steps. (In special cases, when the two points $p$ and $q$ share a coordinate value, the shortest path cannot contain diagonal steps. Thus, the number of shortest weighted paths is exactly one in this case.) Let $i$ be the number of diagonal steps in the shortest path (these steps can be replaced by $\alpha$-steps); then, $i$ has a range between $0 \leq i \leq \min \left\{w_{1}, w_{2}\right\}$. Because each diagonal step can be replaced by two consecutive $\alpha$-steps, we need to sum up the cases, such as the number of shortest weighted paths corresponding to various value of $i$. This can be done as follows:

$i=0$, then all steps in the path are $\alpha$-steps: $\left(\begin{array}{c}w_{1}+w_{2} \\ w_{1}\end{array}\right)$ : vertical and horizontal steps in any order;

$i=1$, then 1 diagonal step and remaining steps are $\alpha$-steps(horizontal and vertical steps, accordingly): $\frac{\left(w_{1}+w_{2}-1\right) !}{1 !\left(w_{1}-1\right) !\left(w_{2}-1\right) !}$;

$i$ in general, the number of steps is $w_{1}+w_{2}-i$ from which $i$ are diagonal, $w_{1}-i$ and $w_{2}-i$ are the number of horizontal and vertical steps. The number of such paths is $\frac{\left(w_{1}+w_{2}-i\right) !}{i !\left(w_{1}-i\right) !\left(w_{2}-i\right) !} ; i=\min \left\{w_{1}, w_{2}\right\}$, (the same formula applies for this special case, as we have used in the case $\alpha<\beta<2 \alpha):\left(\begin{array}{l}\max \left\{w_{1}, w_{2}\right\} \\ \min \left\{w_{1}, w_{2}\right\}\end{array}\right)$.

To sum these numbers up, the number of shortest weighted paths is computed:

$$
\sum_{i=0}^{\min \left\{w_{1}, w_{2}\right\}} \frac{\left(w_{1}+w_{2}-i\right) !}{i !\left(w_{1}-i\right) !\left(w_{2}-i\right) !}
$$

As is shown in the formula, each time we increment $i$ by 1 , the number of diagonal steps is increased by 1 and the number of $\alpha$-steps is decreased by 2 (1 vertical step, 1 horizontal step); then, the overall number of steps in the shortest path is decreased by $i$ for each $i$, where in this shortest path we have $i$ diagonal 
steps, $w_{1}-i$ horizontal steps and $w_{2}-i$ vertical steps. Therefore, the number of shortest weighted paths according to the value of $i$ of diagonal steps is given as $f\left(w_{1}, w_{2}, i\right)=\frac{\left(w_{1}+w_{2}-i\right) !}{i !\left(w_{1}-i\right) !\left(w_{2}-i\right) !}$ using the fact that the order of steps is arbitrary.

Example 4. Let $p(15,1)$ and $q(17,3)$ and weights $\alpha=3, \beta=6$ be given. Then, $w_{1}=2$, $w_{2}=2$ and $\min \left\{w_{1}, w_{2}\right\}=2$, thus, the number of shortest paths is:

$$
f(2,2)=\sum_{i=0}^{2} \frac{(2+2-i) !}{i !(2-i) !(2-i) !}=13 .
$$

As we can see, the number of shortest weighted paths, in this case, can be computed by various numbers of diagonal steps with a maximum of $\min \left\{w_{1}, w_{2}\right\}$. Figure 6 (a), (b) and (c) shows all the possible shortest paths between points $p$ and $q$ of Example 4 separated by the possible number of diagonal steps.

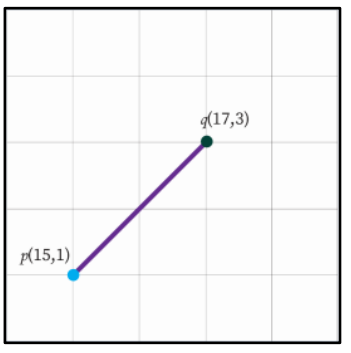

(a)

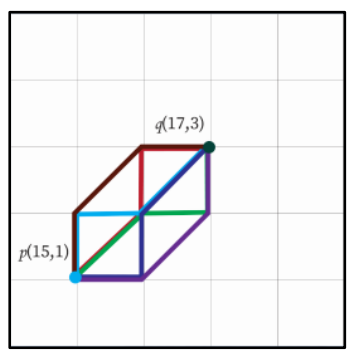

(b)

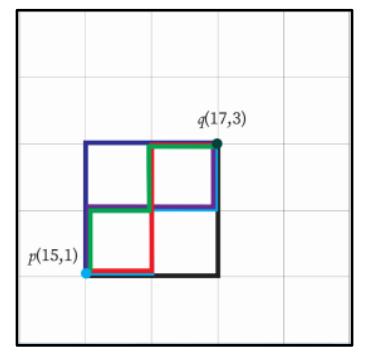

(c)

Figure 6

The shortest paths between $p(15,1)$ and $q(17,3)$, with $\alpha=3$ and $\beta=6$ (case $2 \alpha=\beta>0$ ), when $i=0,1$ and 2 is the number of diagonal steps (starting from $i=0$ to $i=\min \left\{w_{1}, w_{2}\right\}$ in the path from $p$ to $q$

Summarizing the results of this case, Figure 7 shows the 3D chart for the values of the number of shortest weighted paths from point $(0,0)$ to all points in a $14 \times 14$ window with the origin in the middle. The function grows most rapidly on the diagonal directions.

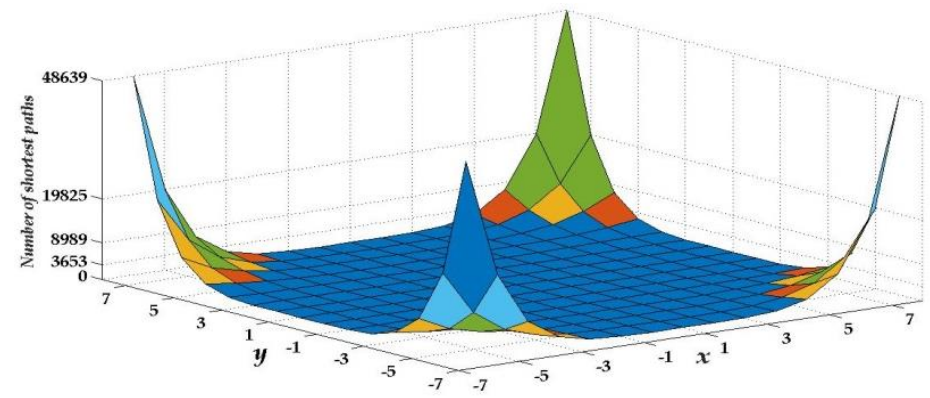

Figure 7

The number of shortest weighted paths from the point $(0,0)$ to other points in a $14 \times 14$ window with corners $(-7,-7),(7,-7),(7,7)$ and $(-7,7)$, in case $\beta=2 \alpha>0$ 


\subsection{Case of $\beta=\alpha>0$}

Theorem 4. Let $\alpha$ and $\beta$ be (positive) weights for cityblock and diagonal movements, respectively, with $\alpha=\beta$. Let $p=\left(x_{1}, y_{1}\right), q=\left(x_{2}, y_{2}\right), w_{1}=\left|x_{1}-x_{2}\right|$ and $w_{2}=$ $\left|y_{1}-y_{2}\right|$. The number $f\left(w_{1}, w_{2}\right)$ of the shortest paths between $p$ and $q$ is counted as

$$
f\left(w_{1}, w_{2}\right)=\sum_{i=0}^{\frac{\max \left\{w_{1}, w_{2}\right\}-\min \left\{w_{1}, w_{2}\right\}}{2}}\left(\begin{array}{c}
\max \left\{w_{1}, w_{2}\right\} \\
i
\end{array}\right)\left(\begin{array}{l}
\max \left\{w_{1}, w_{2}\right\}-i \\
\min \left\{w_{1}, w_{2}\right\}+i
\end{array}\right)
$$

Proof. In this case, the weight of a diagonal step equals the weight of an $\alpha$ step (i.e. a vertical or horizontal step). The number of steps in a shortest path is clearly given by $\max \left\{w_{1}, w_{2}\right\}$ (as in chessboard distance). Since one does not need to pay any extra for diagonal steps, it is possible, for example, that instead of having two consecutive $\alpha$ steps in the same direction, two diagonal steps are applied, reaching the same point after the two steps. In these paths, there are diagonal steps that are in an unnecessary direction (i.e. shortest paths can be obtained without any such direction steps). Let $i$ denote the number of such unnecessary direction diagonal steps. Evidently, the minimum value of $i$ is 0 . For any such step, we need to have an extra diagonal step (in the other diagonal direction, to equalize its effect) instead of an $\alpha$ step. Originally, without any unnecessary diagonal steps $(i=0)$, there are exactly $\max \left\{w_{1}, w_{2}\right\}-\min \left\{w_{1}, w_{2}\right\}$ number of $\alpha$ steps in a shortest weighted path. Thus, the number of $\alpha$ steps decreases by two when an unnecessary diagonal step is introduced. Thus, the maximum of $i$ will be $\left\lfloor\frac{\max \left\{w_{1}, w_{2}\right\}-\min \left\{w_{1}, w_{2}\right\}}{2}\right]$, where the floor function is used. When $i$ is fixed, we know the number of various steps in the shortest path(s): there are $\max \left\{w_{1}, w_{2}\right\}$ steps, from which $i$ are unnecessary diagonal steps, and we have also $\min \left\{w_{1}, w_{2}\right\}+i$ number of diagonal steps in the other diagonal direction. The remaining steps are $\alpha$ steps, and their number is $\left(\max \left\{w_{1}, w_{2}\right\}-i\right)-\left(\min \left\{w_{1}, w_{2}\right\}+i\right)=\max \left\{w_{1}, w_{2}\right\}-\min \left\{w_{1}, w_{2}\right\}-2 i$.

Thus, the number of shortest paths with various values of $i$ can be computed as follows:

$i=0$, then all steps in the path are in the right direction diagonal and $\alpha$-steps, and their number is $\left(\begin{array}{c}\max \left\{w_{1}, w_{2}\right\} \\ \min \left\{w_{1}, w_{2}\right\}\end{array}\right)$;

for $i$ in general:

$\frac{\max \left\{w_{1}, w_{2}\right\} !}{i !\left(\min \left\{w_{1}, w_{2}\right\}+i\right) !\left(\max \left\{w_{1}, w_{2}\right\}-\min \left\{w_{1}, w_{2}\right\}-2 i\right) !}$

Where $i=\left[\frac{\max \{w 1, w 2\}-\min \{w 1, w 2\}}{2}\right]$ is the maximum value for $i$. Thus, the total number of shortest paths is the sum of those:

$f\left(w_{1}, w_{2}\right)=\sum_{i=0}^{\left\lfloor\frac{\max \left\{w_{1}, w_{2}\right\}-\min \left\{w_{1}, w_{2}\right\}}{2}\right.}\left(\begin{array}{c}\max \left\{w_{1}, w_{2}\right\} \\ i\end{array}\right)\left(\begin{array}{l}\max \left\{w_{1}, w_{2}\right\}-i \\ \min \left\{w_{1}, w_{2}\right\}+i\end{array}\right)$ 
Example 5. Let us use the points $p(-18,9)$ and $q(-15,9)$ with weight values $\alpha=1$, $\beta=1$. Then $w_{1}=3, w_{2}=0$ and thus, $\min \left\{w_{1}, w_{2}\right\}=0, \max \left\{w_{1}, w_{2}\right\}=3$. Further, the number of shortest weighted path (where the distance is 3 ) is:

$$
f(3,0)=\sum_{i=0}^{1}\left(\begin{array}{c}
\max \{3,0\} \\
i
\end{array}\right)\left(\begin{array}{c}
\max \{3,0\}-i \\
\min \{3,0\}+i
\end{array}\right)=7
$$

These paths are also illustrated in Figure 8 (a) and (b), with $i=0$ and $i=1$, respectively.

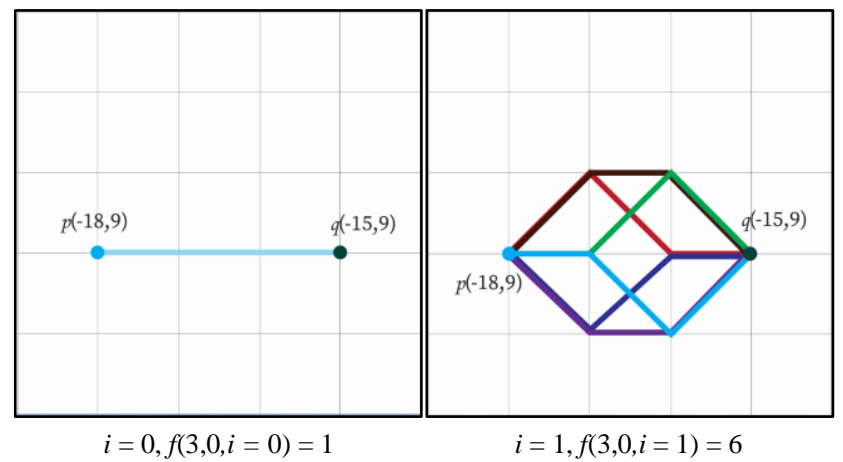

(a)

(b)

Figure 8

Shortest paths between $p(-18,9)$ and $q(-15,9)$ with $\alpha=\beta$, when $i=0$ and 1 , respectively in (a) and (b), where $i$ is the number possible diagonal steps to an unnecessary direction in the path

from $p$ to $q$

To show how these numbers are changing in the function of the coordinate differences, in Figure 9 we present a 3D chart for the number of shortest paths from the origin to other points in a $14 \times 14$ window when the diagonal and cityblock steps have the diagonals at the minimum places of this curve while it grows rapidly on the axes.

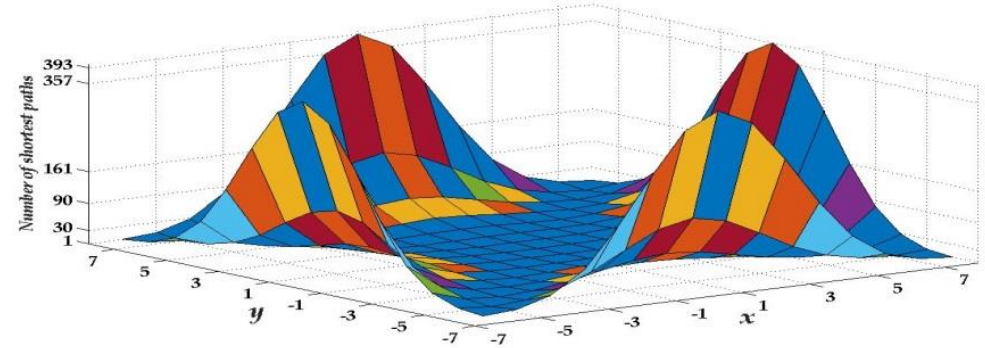

Figure 9

The number of shortest weighted paths from point $(0,0)$ to other points in a $14 \times 14$ window with corners $(-7,-7),(7,-7),(7,7)$ and $(-7,7)$, in case $\beta=\alpha>0$ 


\subsection{Case of $0<\beta<\alpha$}

In this case, $\beta$ steps (diagonal steps) have less weight than $\alpha$ steps (i.e. horizontal and vertical steps); therefore, it will be more convenient and shorter to move from one point to another by diagonal steps, the shortest path between two points relying on the parity of the sum $S$ of the absolute differences of the coordinates of the points. Therefore, we discuss two sub-cases in the two subsections below.

\subsubsection{Sub-Case of $0<\beta<\alpha$ for Points with Even Sum of Differences}

Theorem 5.1. Let $\alpha$ and $\beta$ be the weights for cityblock and diagonal movements, respectively, with $\alpha>\beta$. Let $p=\left(x_{1}, y_{1}\right), q=\left(x_{2}, y_{2}\right), w_{1}=\left|x_{1}-x_{2}\right|$ and $w_{2}=\left|y_{1}-y_{2}\right|$. If $S=w_{1}+w_{2}$ is an even number, then the number of the shortest paths between $p$ and $q$, denoted by $f\left(w_{1}, w_{2}\right)$, is computed as

$f\left(w_{1}, w_{2}\right)=\left(\begin{array}{c}\max \left\{w_{1}, w_{2}\right\} \\ \frac{\min \left\{w_{1}, w_{2}\right\}+\max \left\{w_{1}, w_{2}\right\}}{2}\end{array}\right)$

Proof. The number of steps between two points is given as $\max \left\{w_{1}, w_{2}\right\}$; moreover, all of them can be diagonal steps. As we showed previously in Subsection 3.2 (case $\alpha<\beta<2 \alpha$ ), $\min \left\{w_{1}, w_{2}\right\}$ is the number of original diagonal steps in a shortest path. The remaining number of steps, $\max \left\{w_{1}, w_{2}\right\}-\min \left\{w_{1}, w_{2}\right\}$, can also be expressed by diagonal steps in this case; we call these diagonal steps 'added' diagonal steps. These added diagonal steps are used instead of the $\alpha$-steps of the case $\alpha<\beta<2 \alpha$. These added diagonal steps are of two directions. One of them is the one we have called 'unnecessary' direction. We must have them in this case if $w_{1} \neq w_{2}$. (In case of equality, the shortest path is built up by original diagonal steps to the same direction.) We need to add the same number of unnecessary direction diagonal steps and other (original) direction steps. Thus, the number of unnecessary direction diagonal steps is $\frac{\max \left\{w_{1}, w_{2}\right\}-\min \left\{w_{1}, w_{2}\right\}}{2}$, and the same number of added diagonal steps is needed. Therefore, the number of diagonal steps in a shortest path is $\left(\min \left\{\left\{w_{1}, w_{2}\right\}+\frac{\max \left\{w_{1}, w_{2}\right\}-\min \left\{w_{1}, w_{2}\right\}}{2}\right)+\right.$ $\frac{\max \left\{w_{1}, w_{2}\right\}-\min \left\{w_{1}, w_{2}\right\}}{2}$. The first term gives the number of original direction diagonal steps (both the original and the added ones), while the second term gives the unnecessary direction diagonal steps. The sum equals $\max \left\{w_{1}, w_{2}\right\}$.

Since the order of these steps is arbitrary, the number of shortest weighted paths between points $p$ and $q$ is the number of possible arrangements of these diagonal steps in the shortest path. Consequently, their number can be expressed by the following equation:

$$
f\left(w_{1}, w_{2}\right)=\left(\begin{array}{c}
\max \left\{w_{1}, w_{2}\right\} \\
\frac{\max \left\{w_{1}, w_{2}\right\}+\min \left\{w_{1}, w_{2}\right\}}{2}
\end{array}\right)
$$

Equivalently, it can be written as the following binomial coefficient: 
$f\left(w_{1}, w_{2}\right)=\left(\begin{array}{c}\max \left\{w_{1}, w_{2}\right\} \\ \min \left\{w_{1}, w_{2}\right\}+\frac{\max \left\{w_{1}, w_{2}\right\}-\min \left\{w_{1}, w_{2}\right\}}{2}\end{array}\right)$

Let us analyse a special case. When $w_{1}$ or $w_{2}$ equals zero, the number of original diagonal steps is $\min \left\{w_{1}, w_{2}\right\}=0$, and the shortest path contains only added diagonal steps: one (any) of the directions is then unnecessary, and we have the same number of other added diagonal steps. In this case, the previous formula, the number of shortest weighted paths is simplified as follows:

$$
f\left(w_{1}, w_{2}\right)=\left(\begin{array}{l}
\max \left\{w_{1}, w_{2}\right\} \\
\frac{\max \left\{w_{1}, w_{2}\right\}}{2}
\end{array}\right)
$$

Example 6. Let $p(0,0), q(3,1), \alpha=2$ and $\beta=1$ be given. Then, $w_{1}=|3-0|=3$ and $w_{2}=|1-0|=1$, then the number of shortest paths between $p$ and $q$ is:

$$
f(3,1)=\left(\begin{array}{c}
\max \{3,1\} \\
\frac{\max \{3,1\}+\min \{3,1\}}{2}
\end{array}\right)=\left(\begin{array}{l}
3 \\
2
\end{array}\right)=3
$$

These paths are illustrated in Figure 10.

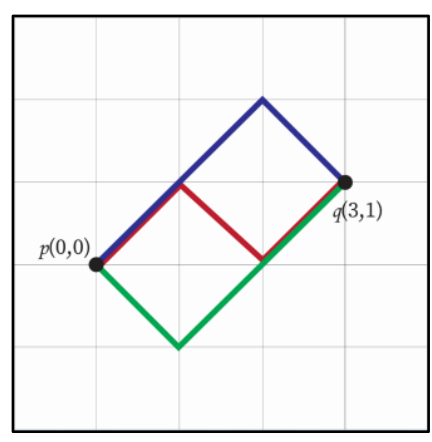

Figure 10

The shortest paths between $p(0,0)$ and $q(3,1)$ in case $0<\beta<\alpha$ with even sum of differences

\subsubsection{Sub-Case of $0<\beta<\alpha$ for Points with Odd Sum of Differences}

Theorem 5.2. Let $\alpha$ and $\beta$ be the weights for cityblock and diagonal movements, respectively, with $\alpha>\beta$. Let $p=\left(x_{1}, y_{1}\right)$ and $q=\left(x_{2}, y_{2}\right)$ be two points in the square grid and $w_{1}=\left|x_{1}-x_{2}\right|$ and $w_{2}=\left|y_{1}-y_{2}\right|$. If $S=w_{1}+w_{2}$ is an odd number, then the number $f\left(w_{1}, w_{2}\right)$ of the shortest paths between the points $p$ and $q$ is determined as

$$
f\left(w_{1}, w_{2}\right)=\left(\begin{array}{c}
\max \left\{w_{1}, w_{2}\right\}-1 \\
\frac{\min \left\{w_{1}, w_{2}\right\}+\max \left\{w_{1}, w_{2}\right\}-1}{2}
\end{array}\right) \cdot \max \left\{w_{1}, w_{2}\right\}
$$

Proof. In this case, we must have a cityblock step ( $\alpha$ step) in the path because there is no way to have a shortest path with only diagonal steps (with $\beta$ steps, two coordinates are always modified by \pm 1 , and thus odd difference cannot be 
eliminated). The number of diagonal steps, thus, is $\max \left\{w_{1}, w_{2}\right\}-1$, since the number of steps in this shortest path is $\max \left\{w_{1}, w_{2}\right\}$. Let $q$ ' be the cityblock neighbour of $q$ that is the closest to $p$ (i.e. a shortest path between $p$ and $q$ ' can be obtained by $\max \left\{w_{1}, w_{2}\right\}-1$ diagonal steps). Actually, a shortest path from $p$ to $q$ contains exactly the same number of various direction steps as the shortest path from $p$ to $q$ ' plus an additional cityblock step in the direction that is the same as from $q$ ' to $q$. The number of shortest paths is counted as the number of possible arrangements of the diagonal steps and the cityblock steps. Applying Theorem 5.1, the number of ways to have the diagonal steps between $p$ and $q$ is as follows:

$$
\left(\begin{array}{c}
\max \left\{w_{1}, w_{2}\right\}-1 \\
\frac{\min \left\{w_{1}, w_{2}\right\}+\max \left\{w_{1}, w_{2}\right\}-1}{2}
\end{array}\right)
$$

Then, the number of ways to locate one cityblock step (which may not necessarily be the last step of the shortest path, but can be anywhere) is as follows:

$$
\left(\begin{array}{c}
\max \left\{w_{1}, w_{2}\right\} \\
1
\end{array}\right)=\max \left\{w_{1}, w_{2}\right\}
$$

From these, the number of shortest weighted paths is given by the following equation:

$$
f\left(w_{1}, w_{2}\right)=\left(\begin{array}{c}
\max \left\{w_{1}, w_{2}\right\}-1 \\
\frac{\min \left\{w_{1}, w_{2}\right\}+\max \left\{w_{1}, w_{2}\right\}-1}{2}
\end{array}\right) \cdot \max \left\{w_{1}, w_{2}\right\}
$$

Example 7. Let the points $p(2,0)$ and $q(4,3)$, and the weights $\alpha=3$ and $\beta=2$ be given. Then $w_{1}=|4-2|=2$ and $w_{2}=|3-0|=3$, therefore $w_{1}+w_{2}=5$ (which is odd number), then the number of shortest paths between $p$ and $q$ is:

$$
f(2,3)=\left(\begin{array}{c}
\max \{2,3\}-1 \\
\frac{\max \{2,3\}+\min \{2,3\}-1}{2}
\end{array}\right) \cdot \max \{2,3\}=\left(\begin{array}{l}
2 \\
2
\end{array}\right) \cdot 3=3
$$

Figure 11 shows all these shortest paths.

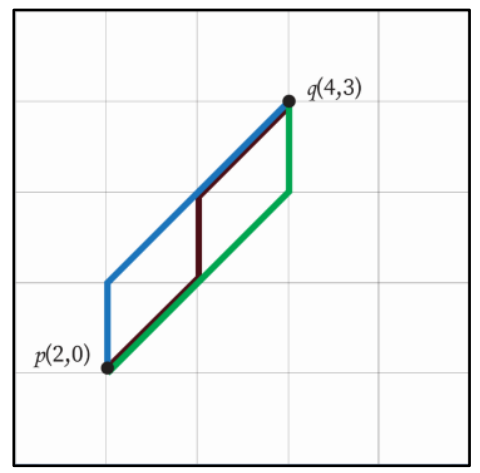

Figure 11

The shortest paths between $p(2,0)$ and $q(4,3)$, in case $0<\beta<\alpha$ with odd sum of differences 


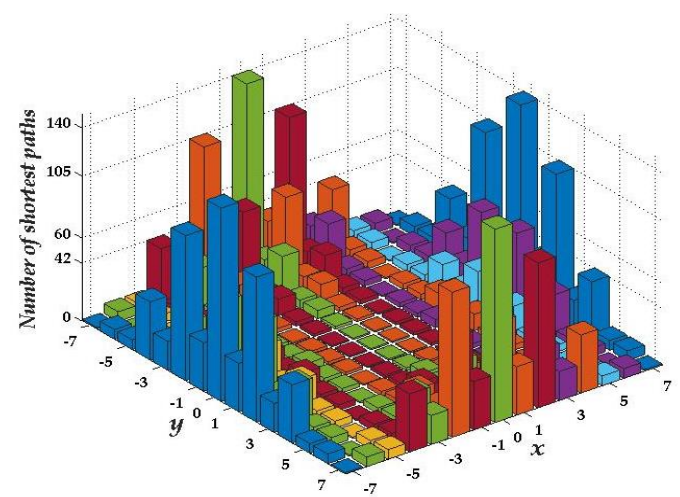

Figure 12

The number of shortest weighted paths from the origin $(0,0)$ to other points in a $14 \times 14$ window with corners $(-7,-7),(7,-7),(7,7)$ and $(-7,7)$, in case $0<\beta<\alpha$.

Finally, for summarizing the case when diagonal steps have lower weights than cityblock steps, Figure 12 shows the number of shortest paths between $(0,0)$ and other points in a $14 \times 14$ window. For the subcases, we also separately show the values: Figure 13 represents the cases $(\beta<\alpha$ for points with even coordinate sum $S)$ and $(\beta<\alpha$ for points with odd coordinate sum $S)$ for the number of shortest weighted paths from point $(0,0)$ in a $14 \times 14$ window. One can observe that minimal values are given on the diagonals, while the function is growing with different speeds for the points with odd and even coordinate sums. For odd coordinate sums, it grows more rapidly. The largest growth values are on the axes with a growing coordinate difference.
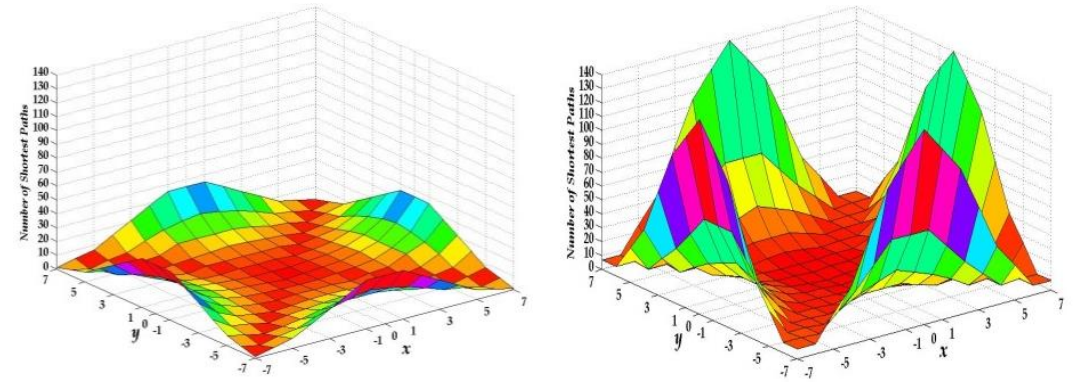

Figure 13

The number of shortest weighted paths from the origin $(0,0)$ to other points in a $14 \times 14$ window with corners $(-7,-7),(7,-7),(7,7)$ and $(-7,7)$, in case $\beta<\alpha$ for points with even coordinate sum $\mathrm{S}$ shown on the left and with odd coordinate sum $\mathrm{S}$ shown on the right 


\subsection{Cases with Zero Weights}

In this subsection we consider the special scenarios when one or both of the weights is/are 0 . In subsection 3.6.1 we consider the cases when $\alpha=0$, while in 3.6.2 we consider the case when $\alpha$ is positive, but $\beta$ has zero value. Up to our knowledge, these cases were never considered before.

\subsubsection{Case of $\alpha=0$}

Theorem 6.1. Let $\alpha=0$ and $\beta \geq 0$ be the weights for cityblock and diagonal movements, respectively. The distance of points $p=\left(x_{1}, y_{1}\right)$ and $q=\left(x_{2}, y_{2}\right)$ is 0 , since there are paths between any two points built up only by cityblock steps. Moreover, there are infinitely paths between $p$ and $q$ with sum of the weights 0 .

Proof. Consider the path built up by cityblock steps along the line from $p=\left(x_{1}, y_{1}\right)$ to $r=\left(x_{1}, y_{2}\right)$ with fixed first coordinate concatenated with the path from $r$ to $q=$ $\left(x_{2}, y_{2}\right)$ on the line with fixed second coordinate. (One or both of these paths could be empty, i.e., with 0 steps, depending on the fact if the points $p=\left(x_{1}, y_{1}\right)$ and $p=$ $\left(x_{1}, y_{1}\right)$ share one or two or no coordinates.) The cost of this path is 0 and thus, the distance of the points with the condition $\alpha=0$ is also zero.

Now, w.l.o.g., assume that $x_{1} \leq x_{2}$. Let us consider the paths defined as follows: cityblock steps along the lines from $p=\left(x_{1}, y_{1}\right)$ to $p^{\prime}=\left(x_{1}-n, y_{1}\right)$ (for any positive integer $n)$, then from $p^{\prime}$ to $r^{\prime}=\left(x_{1}-n, y_{2}\right)$, and from $r$ ' to $q$. Since the sum of the weights of this path is 0 for any value of $n$, all of these paths are considered as shortest paths between the two mentioned points, thus, there are infinitely many of them.

Theorem 6.2. Let $\alpha=0$ and $\beta \geq 0$ be the weights for cityblock and diagonal movements, respectively. The weighted distance defined by these weights is not metrical, but it is a pseudometric.

Proof. A pseudometric is a distance function which has non-negative values, is symmetric, fulfils the triangular inequality, and such that the distance from any point to itself is 0 . All of these properties are easy to check since all distance values are 0 . A distance is metric if it is a pseudometric, moreover if the distance of two points is 0 , then the points coincide. This additional property is dropped by the considered distance function, thus it is not a metric.

Actually, the given pseudometric is the trivial pseudometric, since all the distance values are zero.

\subsubsection{Case of $\alpha>\beta=0$}

Theorem 6.3. Let $\beta=0$ and $\alpha>0$ be the weights for cityblock and diagonal movements, respectively. The distance of points $p=\left(x_{1}, y_{1}\right)$ and $q=\left(x_{2}, y_{2}\right)$ is 0 if and only if the sum of coordinate differences, $w_{1}+w_{2}=\left|x_{1}-x_{2}\right|+\left|y_{1}-y_{2}\right|$ is even. On the other hand, the distance of points $p$ and $q$ is $\alpha$ if and only if the sum of 
coordinate differences, $w_{1}+w_{2}$ is odd. The number of paths between the points with the given length is infinite in both cases.

Proof. Consider, first, the case, when the sum of the coordinate differences is even. There are paths between any two points built up only by diagonal steps. For instance, consider the diagonal line with slope 1 containing point $p$ and the "antidiagonal" line, the line with slope -1 going through on $q$. These two lines will intersect each other at a point $r$ with coordinates $x_{1}+n=x_{2}-m$ and $y_{1}+n=$ $y_{2}+m$ for a pair of integers $n$ and $m$, where these integers give the number (and the direction) of the diagonal steps from $p$ to $r$ and from $r$ to $q$, respectively. Thus, it is clear that the distance of the points becomes zero. Furthermore, the given path can be easily modified to contain more and more diagonal steps (in a similar manner as we have shown in the proof of Theorem 6.1), thus the number of paths with length 0 becomes infinite.

Now, let us consider the case when the sum of the coordinate differences is an odd number. Since in every diagonal step, both of the coordinates change by \pm 1 , we cannot reach from one (of the points $p$ and $q$ ) the other point only by diagonal steps. However, we can reach any of its cityblock neighbours by only diagonal zero-weight steps, thus, we need one extra cityblock step in the path resulting in the distance of the points being $\alpha$ in this case. As the number of zero length paths between $p$ and a given cityblock neighbour of $q$ is (according to the first part of the proof) is infinite, each of them produces a shortest, i.e., $\alpha$ length path between $p$ and $q$ by adding the last cityblock step, hence the proof.

By a similar proof as the proof of Theorem 6.2, one can also establish the following result.

Theorem 6.4. Let $\alpha>0$ and $\beta=0$ be the weights for cityblock and diagonal movements, respectively. The weighted distance defined by these weights is not metrical, but it is a pseudometric.

Finally, in the last subsection, we consider the case, when at least one of the weights is negative.

\subsection{Case of Negative Weight(s)}

Table 1

The discussed cases for the weights $\alpha$ and $\beta$

\begin{tabular}{|c|c|c|c|c|c|c|c|}
\hline \multirow{2}{*}{ Condition } & \multicolumn{5}{|c|}{ On $1 \mathrm{y}$ p o s i t ive w e ig h t s } & & \\
\cline { 2 - 9 } & $2 \alpha<\beta$ & $2 \alpha=\beta$ & $\alpha<\beta<2 \alpha$ & $\alpha=\beta$ & $\beta<\alpha$ & $\alpha=0 \leq \beta$ & $\alpha>\beta=0$ \\
\hline Case /subsection & $\mathbf{3 . 1}$ & $\mathbf{3 . 3}$ & $\mathbf{3 . 2}$ & $\mathbf{3 . 4}$ & $\mathbf{3 . 5}$ & $\mathbf{3 . 6 . 1}$ & $\mathbf{3 . 6 . 2}$ \\
\hline
\end{tabular}

If one or both of the weights $\alpha$ and $\beta$ are negative, then it is easy to see that one can find a path between any two points with sum of the weights that is less than any number. In this case the "distance" of the points does not exist, i.e., it could be 
written as $-\infty$. Since such a path (with finite length) does not exist, there is no shortest path. Actually, in these cases the obtained weighted distance is not distance since the condition of the non-negativeness of its value does not hold. With this note we have finished checking all possible cases, and we give a short summary and discussion in the last section.

\section{Discussion and Conclusion}

In fact, our shortest paths can be represented by trajectories on the digital grid. A combinatorial problem, the number of cheapest solutions, i.e., the number of shortest weighted paths is computed in various scenarios. The numbers of shortest paths with the cityblock and chessboard metrics were already known [6]. However, we have presented results for a much larger class of digital distances, for chamfer distances, in this way our study can be seen as a generalisation of these previous results. Digital distances can be used in various ways in communication networks [16], and they are also related to combinatorial problems. For example, the number of shortest paths gives important features of a network. Results on the number of shortest paths for neighbourhood sequence distances were presented in [15], in this sense we have completed the picture by presenting here analogous results for the other type of widely used digital distance family. In this paper, we have analysed rigorously all the cases to find the number of minimum weighted paths between any two points in a square grid. The cases depend on the value of weights given to cityblock steps ( $\alpha$ steps) and diagonal steps ( $\beta$ steps). We have discussed five cases with positive weights and two cases when weight zero is allowed. We have seen that the results obtained in them are pairwise different. By Table 1 one can also be sure that there are no more cases, all the possibilities to have positive and/or zero weights for both cityblock and diagonal steps are discussed. Our results with positive weights are also displayed in $3 \mathrm{D}$ graphs, which show how the resulting functions grow. In most cases. the functions have strong monotonic behaviour as one goes further from the origin. The cases of $\beta>2 \alpha$ and $\beta=2 \alpha$ show very similar behaviour (see Figure 4 and 8). The case of $\alpha<\beta<2 \alpha$ does not seem to relate to any other cases, (Figure 6). Case of $\beta=\alpha$, displayed in Figure 10, shows some relation to the case of $\beta<\alpha$, however, this latter is more complicated than the others, see Figure 13. We highlight the results of this letter case, i.e., when the diagonal steps have less weight than cityblock steps. As we have seen, the result is described by two different functions depending on the parity of the sum of the coordinate differences of the points, thus it does not behave in a monotonic way. We have shown also the cases when zero weight is allowed. If the cityblock step has zero weight, all the distances become 0 . Contrary, if only the diagonal movements without cost, but the cityblock steps have a positive weight, then somewhat similarly to the case of $\beta<\alpha$, the result is not monotonous but given by two 
different values alternating for the points of the grid. We have also complemented our results by providing the case when negative weights are allowed. In those cases, we cannot really refer to distances based on the smallest weighted paths. Our results are useful in network analysis, in digital image processing and in shape analysis. We believe that it is important also for the application point of view to consider all the possible cases depending on the possible values of the weights. The number of shortest weighted paths between points that contain a given point or a set of given points can be discussed in the future. For example, if we have path $s, \ldots, b, \ldots, t$, then it can be computed how many shortest paths between $s$ and $t$ contain $b$. Extensions to higher dimensional or other grids (architectures) can also be done.

\section{References}

[1] Alzboon, L., Khassawneh, B., Nagy, B. (2017) On the Number of Weighted Shortest Paths in the Square Grid. Proceedings IEEE $21^{\text {st }}$ Int. Conf. Intelligent Engineering Systems (INES), Larnaca, Cyprus, pp. 83-90, DOI: 10.1109/INES.2017.8118533

[2] Borgefors, G. (1986) Distance transformations in digital images. Computer vision, graphics, and image processing, 34(3), 344-371, DOI: 10.1016/s0734-189x(86)80047-0

[3] Cheng, E., Grossman, J., Qiu, K., Shen, Z. (2013) The number of shortest paths in the arrangement graph. Information Sciences, 240, 191-204, DOI: 10.1016/j.ins.2013.03.035

[4] Das, P., Chakrabarti, P., Chatterji, B. (1987) Distance functions in digital geometry. Information Sciences, 42(1), 113-136, DOI:10.1016/00200255(87)90019-3

[5] Das, P., Chakrabarti, P., Chatterji, B. (1987) Generalized distances in digital geometry. Information Sciences, 42(1), 51-67, DOI: 10.1016/00200255(87)90015-6

[6] Das, P. (1991) Counting minimal paths in digital geometry. Pattern Recognition Letters, 12(10), 595-603, DOI: 10.1016/0167-8655(91)90013$\mathrm{c}$

[7] Das, P. P. (1989) An algorithm for computing the number of the minimal paths in digital images. Pattern Recognition Letters, 9(2), 107-116, DOI: 10.1016/0167-8655(89)90043-3

[8] Hanneman, R., Riddle, M. Introduction to social network methods, Riverside, CA, University of California, Riverside, 2005 (published in digital form at http://faculty.ucr.edu/ hanneman/ [accessed 03.03.2019])

[9] Kari, L., Konstantinidis, S., Sosik, P. (2004) Substitutions, trajectories and noisy channels. CIAA, Kingston, Canada, LNCS, 3317, 2004, 202-212, ISBN: 3-540-24318-6, 978-3-540-24318-2 
[10] Kari, L., Konstantinidis, S., Sosik, P. (2005) Operations on trajectories with applications to coding and bioinformatics. International Journal of Foundations of Computer Science, 16(3), 531-546, DOI: 10.1142/S0129054105003145

[11] Klette, R., Rosenfeld, A. Digital geometry: Geometric methods for digital picture analysis; Morgan Kaufmann, 2004, ISBN: 1-55860-861-3, 978-155860-861-0

[12] Mohanty, G. Lattice Path Counting and Applications, Academic Press, 1979, ISBN: 978-0-12-504050-1

[13] Nagy, B. (2002) Metrics based on neighbourhood sequences in triangular grids. Pure Math. Appl., 13(1), 259-274

[14] Nagy, B. (2003) Distance functions based on neighbourhood sequences. Publ. Math., 63(3), 483-493

[15] Nagy, B. (2015) On the number of shortest paths by neighborhood sequences on the square grid. Joint Austrian-Hungarian Math Conf, Széchenyi István Univ, Győr, Hungary (abstract)

[16] Nagy, B. (2017) Application of neighborhood sequences in communication of hexagonal networks. Discrete Applied Mathematics, 216, 424-440, DOI: 10.1016/j.dam.2015.10.034

[17] Oyama, T., Morohosi, H. (2004) Applying the shortest-path-counting problem to evaluate the importance of city road segments and the connectedness of the network-structured system. International Transactions in Operational Research, 11, 555-573, DOI: 10.1111/j.14753995.2004.00476.x

[18] Rosenfeld, A., Pfaltz, J. (1968) Distance functions on digital pictures. Pattern Recognition, 1(1), 33-61, DOI: 10.1016/0031-3203(68)90013-7

[19] Yamashita, M., Ibaraki, T. (1986) Distances defined by neighborhood sequences. Pattern Recognition, 19(3), 237-246, DOI: 10.1016/00313203(86)90014-2 